Reinhard Heun ${ }^{1 *}$

\title{
A systematic review on the effect of Ramadan on mental health: minor effects and no harm in general, but increased risk of relapse in schizophrenia and bipolar disorder
}

'University of Bonn, Germany

*email: globalpsychiatrylagmx.com

DOI: $10.2478 / g p-2018-0002$

Received: 26 October 2017; Accepted: 26 December 2017

\begin{abstract}
Objectives: Globally, Moslems are the second largest religious group. During the month of Ramadan from dawn to sundown, healthy Moslems are required to refrain from eating, drinking, smoking, sexual activity and harmful behaviour towards others and themselves. Thus Ramadan may change individual physical states and social interactions. Both might affect mental health within society. Consequently, this systematic review looks at the various effects of Ramadan on mental health.

Methods: A literature search on Ramadan and mental health initially identified 294 papers. We finally selected all 22 relevant papers covering Ramadan and mental health from which study data were extracted.

Results: Relevant papers focussed on the general population and healthy volunteers, on subjects practising sports, on subjects with severe physical disorders, on subjects at risk of eating disorders and on subjects with mental health disorders. The effects of Ramadan on mental well-being were mixed. Positive and negative effects were usually minor, except in subjects with schizophrenia and metabolic syndrome, and in subjects with bipolar disorder who suffered a substantial increase of relapses.

Conclusion: Ramadan fasting is safe in most conditions and disorders, but caution is required in subjects with schizophrenia and bipolar disorder. The research on mental health and Ramadan would profit from larger studies with more representative samples to help understand the intra-individual and social factors that affect the mental health and well-being in patients and in society. The scientific potential of such studies may have been overlooked in the psychiatric community.
\end{abstract}

\section{Keywords}

Systematic review, Ramadan, fasting, mental health, depression, anxiety, schizophrenia, bipolar disorder

\section{INTRODUCTION}

\section{Relevance of Ramadan}

Ramadan is the ninth month of the Islamic lunar calendar, referred to as the Holy Month. Fasting during Ramadan is one of the five religious duties of adult Moslems. Moslems who participate will abstain from eating, drinking and smoking during the day from sunrise to sundown. During Ramadan, all wrongdoings including self-destructive behaviour and anger towards others are prohibited. Moslems with severe diseases can refrain from fasting during Ramadan. However, it may not be clear to doctors who is fit or unfit for fasting in different diseases.

After sundown and during the night, Moslems can eat and drink as much as they need, thus compensating for the lack of fluid and calorie intake during abstinence. Late eating and drinking either alone or in social gatherings will reduce the time available for night time sleep. Thus, the body metabolism and the diurnal sleep cycle are likely to be affected by Ramadan (Brahammam 2006).

There are 1.6 billion Moslems in the world living in many different countries and environments. Ramadan thus affects a large proportion of the global population. The social, religious and mental activities and effects of Ramadan may vary by country (Waterhouse et al. 2008).

\section{Ramadan, sleep, fasting and mental health}

Ramadan may affect sexual function in men (Talib et al. 2015). Ramadan can affect the chronobiology of the body temperature and energy expenditure (Bahammam et al. 
2010). The effect of Ramadan fasting on sleep and daytime fatigue may depend on the season of the year, the latitude of the place, and thus the length of day and night. It may also depend on social factors such as working hours, shop opening times, meal and prayer times (Bahammam 2006).

Sleep has been shown to be affected in many psychiatric disorders: Patients with depression often suffer interrupted sleep and sleep-onset rapid eye movement periods. Poor sleep may be an early sign or a trigger of depression and manic episodes in bipolar disorder. Sleep deprivation has been used as a therapy for severe depressive disorders. During manic episodes, the need for sleep is known to be reduced. Temporary sleep changes have been observed during Ramadan (Leiper et al 2008). Most of the effects of Ramadan fasting on sleep can be reproduced by experimental fasting outside Ramadan (BaHammam et al. 2014).

Change of appetite is a main feature of different eating disorders and depression. Excessive fasting is the characteristic of anorexia nervosa. Fasting has been assumed to improve the mood, in general, but has only rarely been used to improve the mood in depression. Such interventions have not become general practice for practical reasons, but the long-term effects of fasting on mood are also not yet clear (Fond et al. 2012).

The present systematic review will focus on mental health and assess how much it is affected by Ramadan. It investigates if Ramadan and the induced fasting have positive or negative effects on mental health in the general population, in special subgroups of the populations and in different patient groups.

\section{METHODS}

\section{Systematic review of the literature}

We performed a literature search on Pubmed and Medline in September 2017 using the following search terms: Ramadan and mental health or depression or anxiety or schizophrenia or bipolar disorder.

The search identified a total of 294 papers. We then reviewed their titles and abstracts.

One paper in French was excluded.

Ninety-nine papers were falsely identified because one of the authors had the name Ramadan, but were then excluded as these papers did not relate to the holy month of Ramadan, or mental health and Ramadan.

Of the remaining 194 papers of which the abstracts were screened, 162 papers did not focus on mental health issues according to the abstracts.

Thirty-two full papers were reviewed and assessed, and one additional paper was identified via the reference list.

Two review papers and one editorial did not contain any data. Eight other papers did not focus on mental health or provide any data on mental health in Ramadan.

The data from 22 studies were extracted and summarised in a table.

\section{Extraction of information}

The data extraction focused on study question, study design, subject characteristics, assessment instruments, outcome, study results, conclusions and possible bias. The foci of individual studies and their designs showed considerable variety. However, we did not apply any further selection of papers concerning study type, quality and outcome, as this would reduce the number of papers considerably and would, therefore, have restricted the current already limited overview on the effects of Ramadan on mental health.

\section{RESULTS}

Table 1 gives the results of paper selection and data extraction. The literature in relation to the effects of Ramadan on mental health is limited to a few areas: that is, effects in the general population and healthy volunteers (7 papers), in sports (3 papers), on eating and eating disorders (3 papers), on severe physical disorders ( 3 papers) and on mental disorders including bipolar disorder and schizophrenia (6 papers).

\section{Effect in general population and healthy subjects}

The studies on the effects of Ramadan on mood and mental health in the general population and healthy volunteers provide contradicting evidence. Three studies reported positive effects: Depression and stress levels, were significantly reduced in Iranian nurses after in comparison with before the holy month of Ramadan; the effects on anxiety levels were lower and statistically non-significant (Koushali et al. 2013). Mood and the processing of emotional stimuli in volunteers 


\begin{tabular}{|c|c|c|c|c|c|c|}
\hline 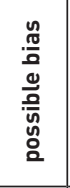 & 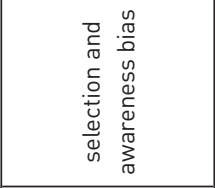 & 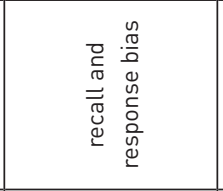 & 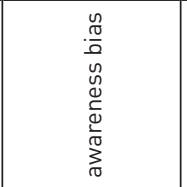 & 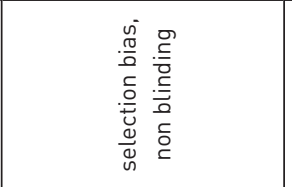 & 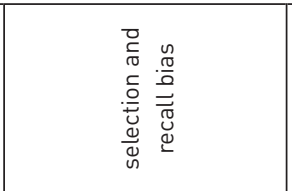 & 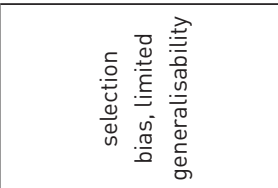 \\
\hline 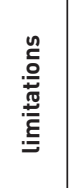 & 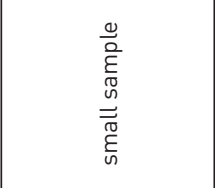 & 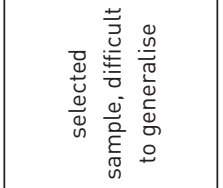 & 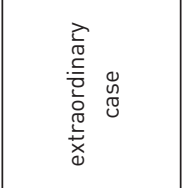 & 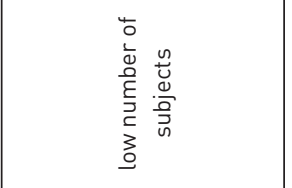 & 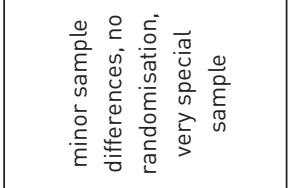 & 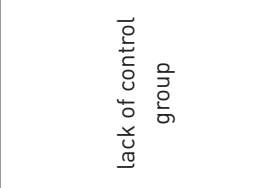 \\
\hline 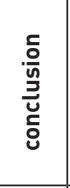 & 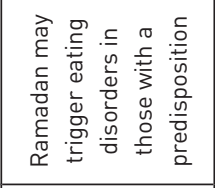 & 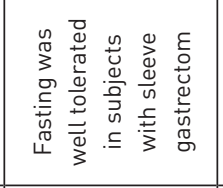 & 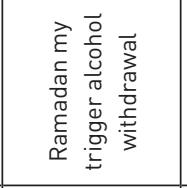 & 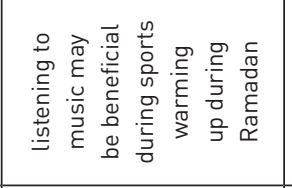 & 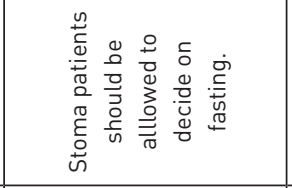 & 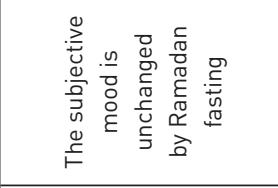 \\
\hline 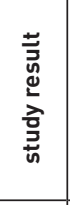 & 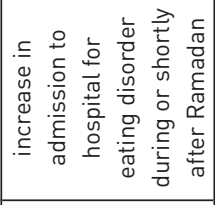 & 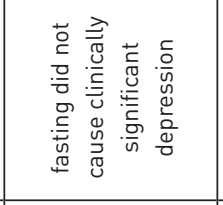 & 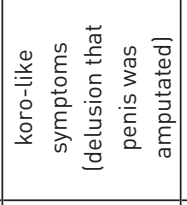 & 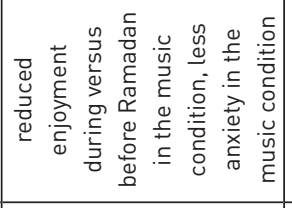 & 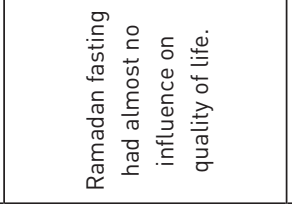 & 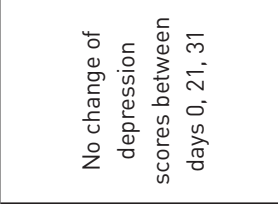 \\
\hline $\begin{array}{l}\check{E} \\
\stackrel{\mathscr{\Xi}}{\Xi}\end{array}$ & 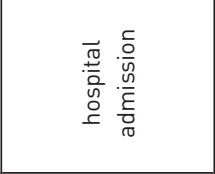 & 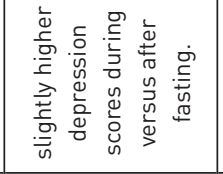 & 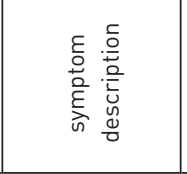 & 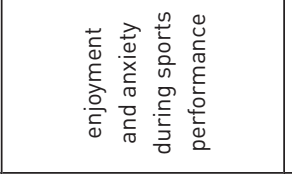 & 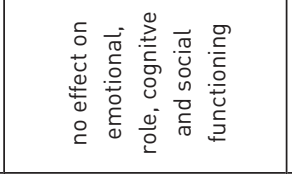 & 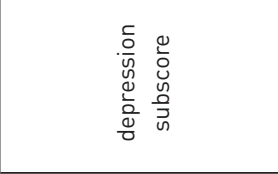 \\
\hline 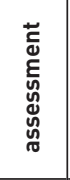 & 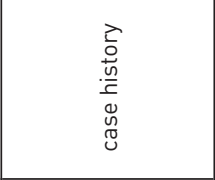 & 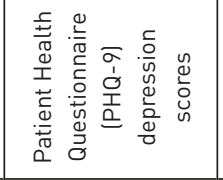 & 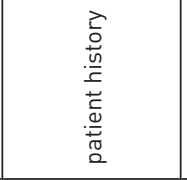 & 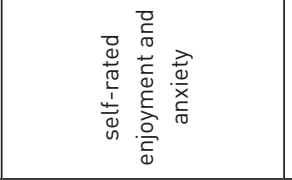 & 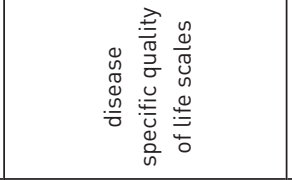 & 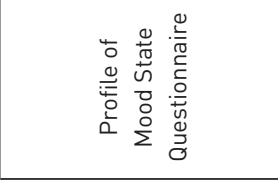 \\
\hline 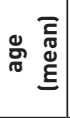 & 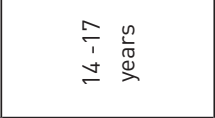 & 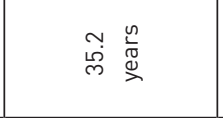 & 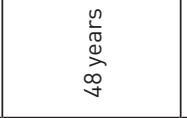 & $\vec{\sim}$ & 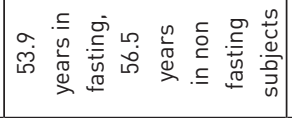 & 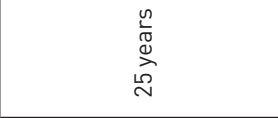 \\
\hline 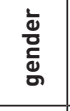 & 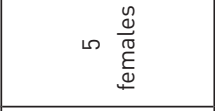 & 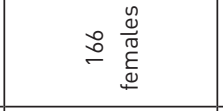 & 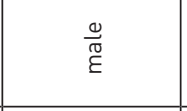 & $\stackrel{\frac{0}{\sigma}}{\frac{\pi}{\varepsilon}}$ & 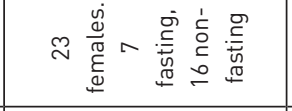 & $\frac{\stackrel{\varrho}{\oplus}}{\stackrel{\oplus}{\varepsilon}}$ \\
\hline 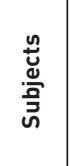 & 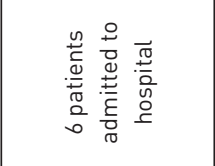 & 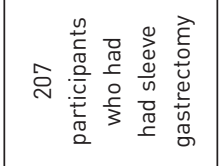 & 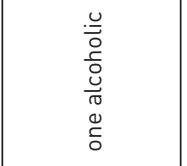 & 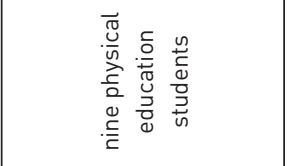 & 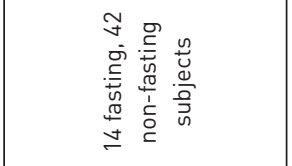 & 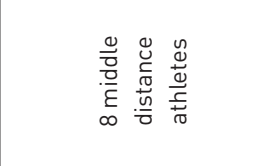 \\
\hline 总 & 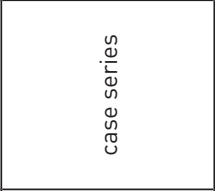 & 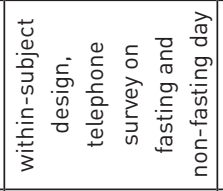 & 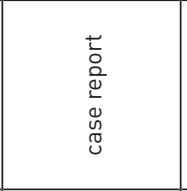 & 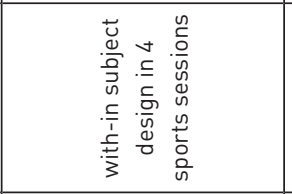 & 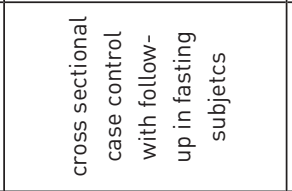 & 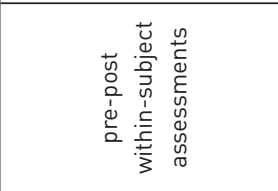 \\
\hline 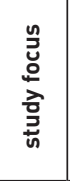 & 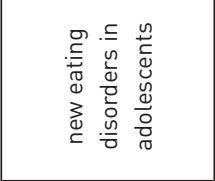 & 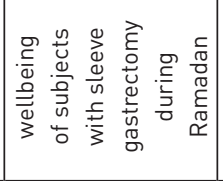 & 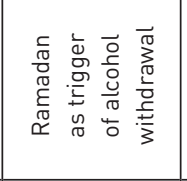 & 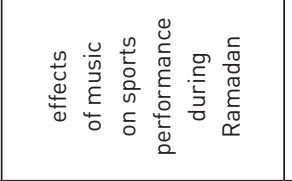 & 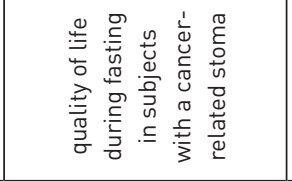 & 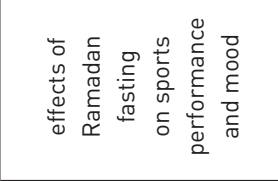 \\
\hline 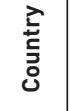 & $\begin{array}{l}\text { बे } \\
\text { 咅 }\end{array}$ & 范 & $\begin{array}{l}\stackrel{\tau}{\tilde{\sigma}} \\
\tilde{\xi}\end{array}$ & $\frac{. \frac{0}{0}}{\stackrel{\frac{5 n}{5}}{5}}$ & $\begin{array}{l}\text { बे } \\
\text { 旁 }\end{array}$ & 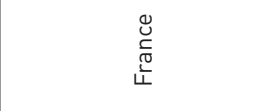 \\
\hline 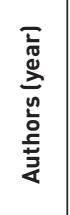 & 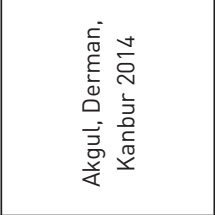 & 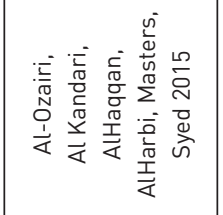 & 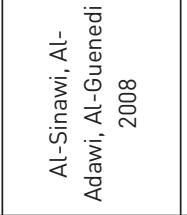 & 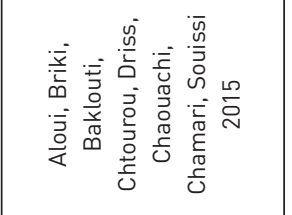 & 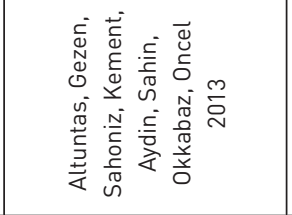 & 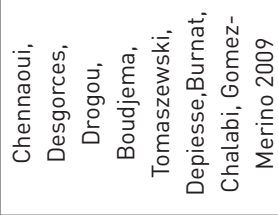 \\
\hline
\end{tabular}




\begin{tabular}{|c|c|c|c|c|c|}
\hline 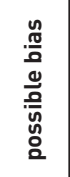 & 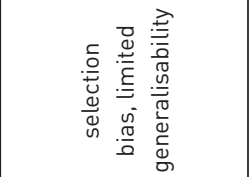 & 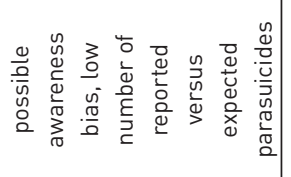 & 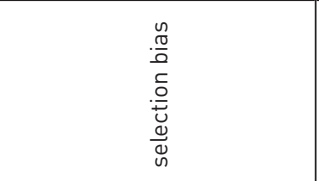 & 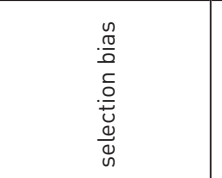 & 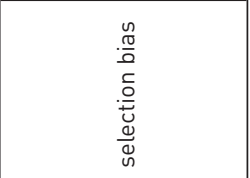 \\
\hline 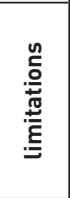 & 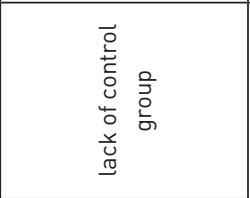 & 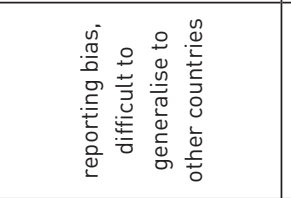 & 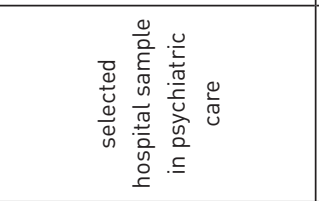 & 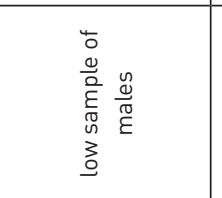 & 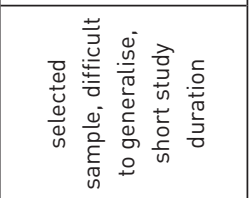 \\
\hline $\begin{array}{l}\frac{c}{\underline{n}} \\
\overline{\underline{u}} \\
\check{c}\end{array}$ & 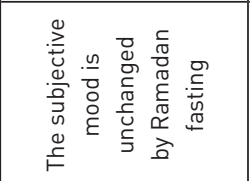 & 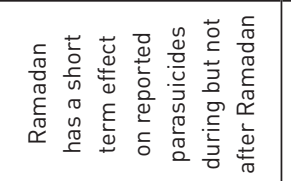 & 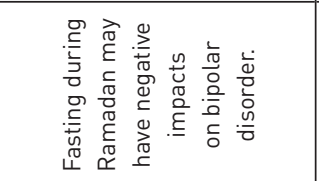 & 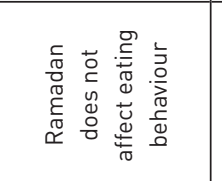 & 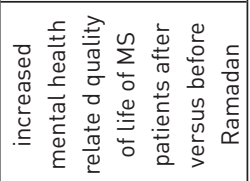 \\
\hline 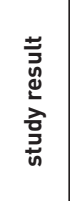 & 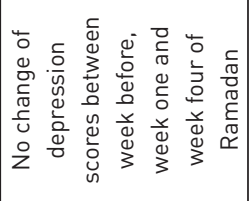 & 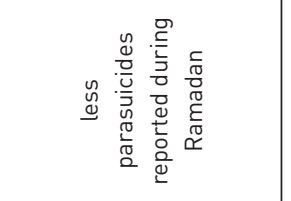 & 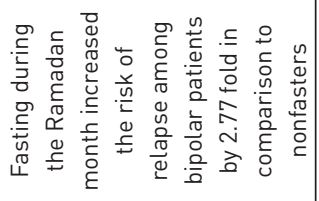 & 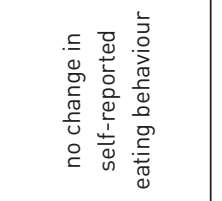 & 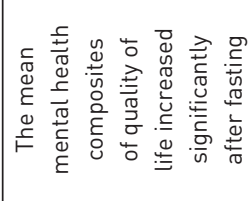 \\
\hline $\begin{array}{l}\stackrel{\Xi}{E} \\
\stackrel{\mathscr{U}}{\Xi} \\
\end{array}$ & 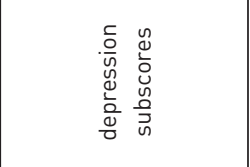 & 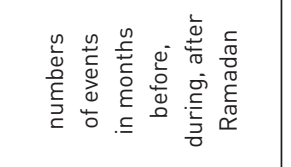 & 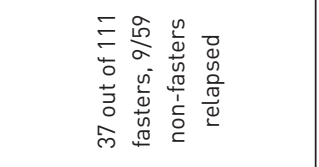 & 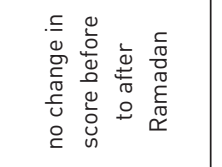 & 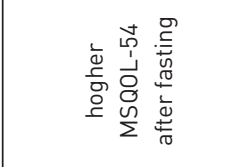 \\
\hline 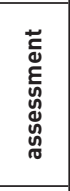 & 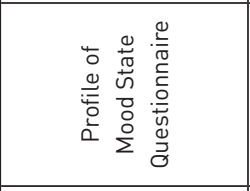 & 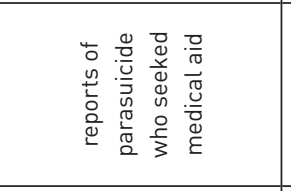 & 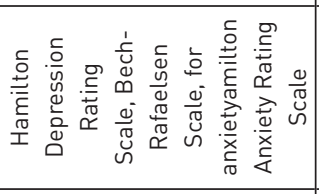 & 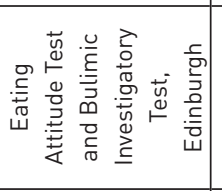 & 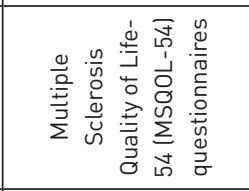 \\
\hline 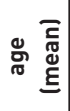 & 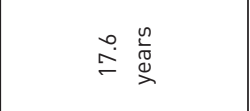 & 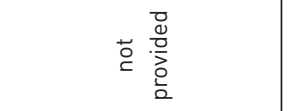 & 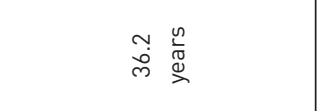 & 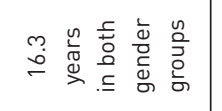 & 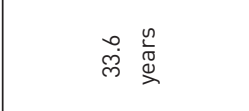 \\
\hline 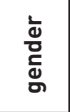 & $\stackrel{\frac{0}{\pi}}{\frac{\pi}{\varepsilon}}$ & 产 & 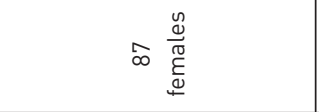 & 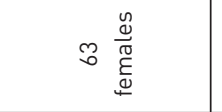 & 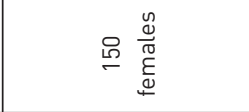 \\
\hline 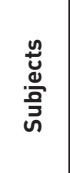 & 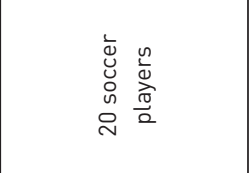 & 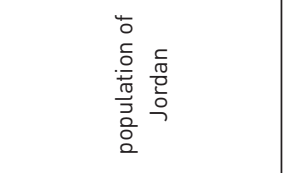 & 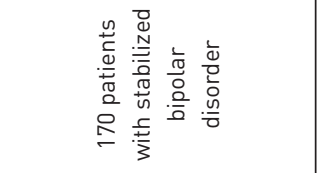 & 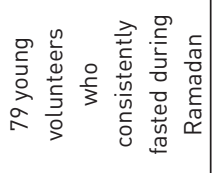 & 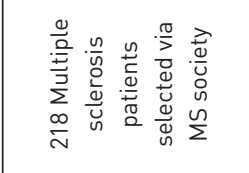 \\
\hline 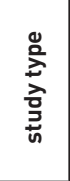 & 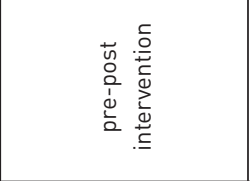 & 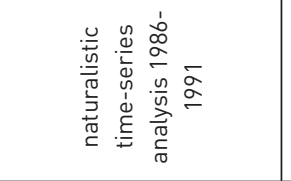 & 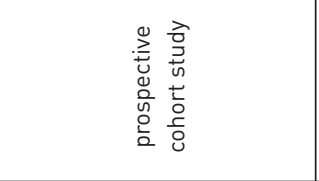 & 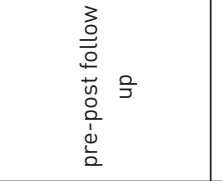 & 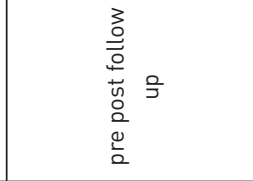 \\
\hline 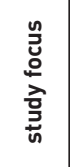 & 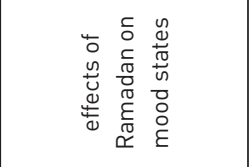 & 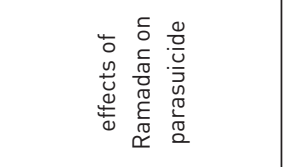 & 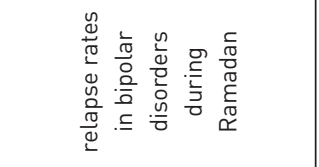 & 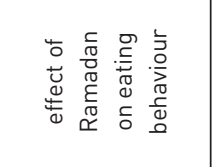 & 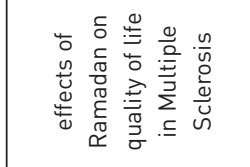 \\
\hline $\begin{array}{l}\text { 吾 } \\
\text { 竞 } \\
\end{array}$ & $\frac{\cdot \frac{0}{n}}{\frac{n}{5}}$ & 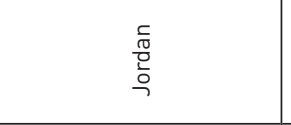 & $\begin{array}{l}\stackrel{̊}{0} \\
\text { ò } \\
\Sigma\end{array}$ & 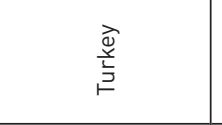 & $\stackrel{\text { 듀 }}{\underline{\underline{5}}}$ \\
\hline 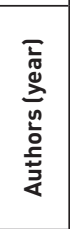 & 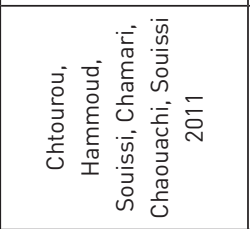 & 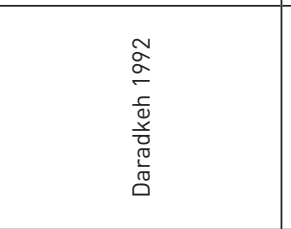 & 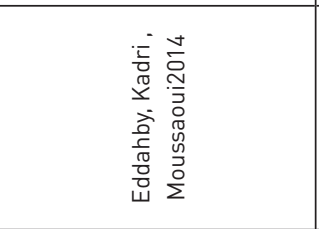 & 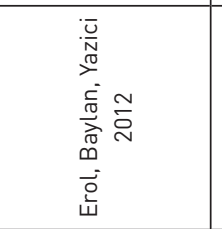 & 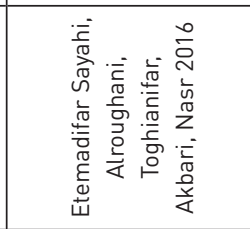 \\
\hline
\end{tabular}




\begin{tabular}{|c|c|c|c|c|c|c|}
\hline 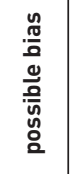 & 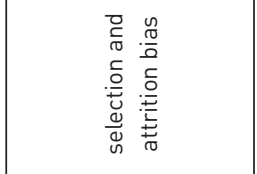 & 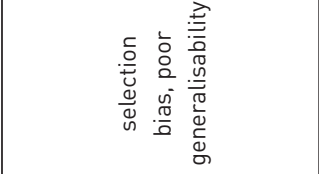 & 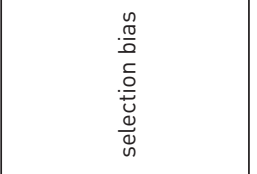 & 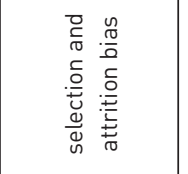 & 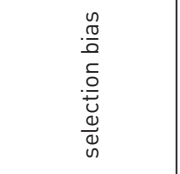 & 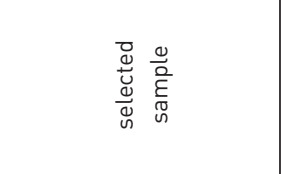 \\
\hline 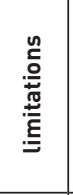 & 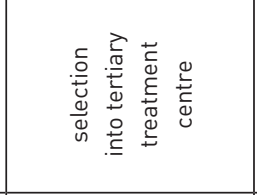 & 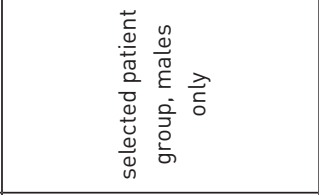 & 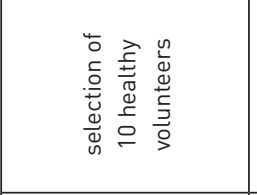 & 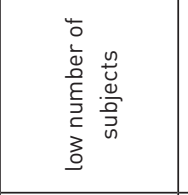 & 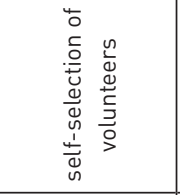 & 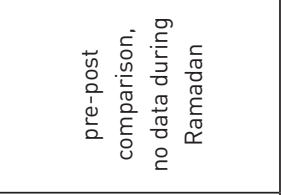 \\
\hline 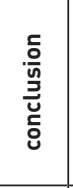 & 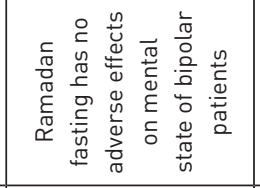 & 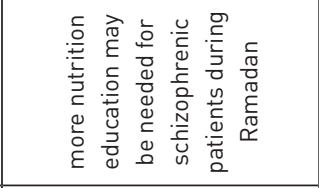 & 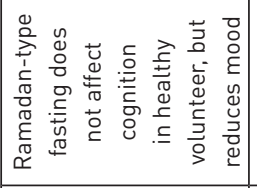 & 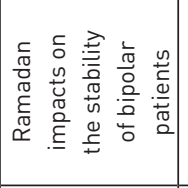 & 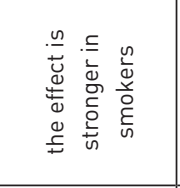 & 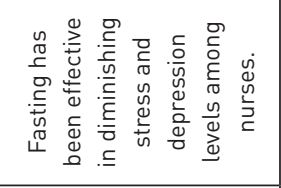 \\
\hline 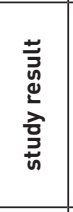 & 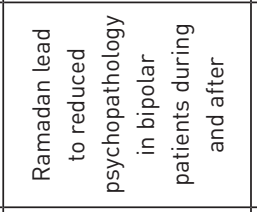 & 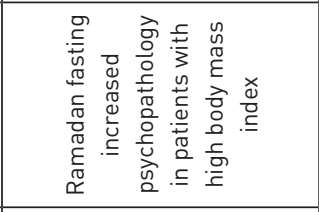 & 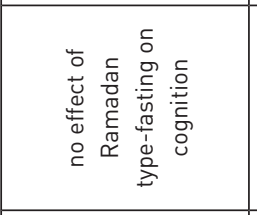 & 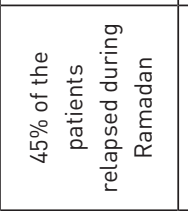 & 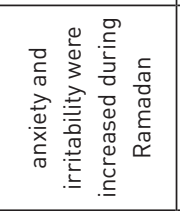 & 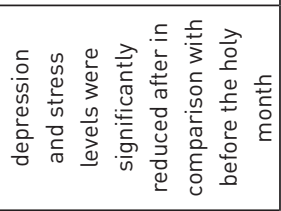 \\
\hline $\begin{array}{l}\mathscr{E} \\
\stackrel{\mathscr{\Xi}}{\Xi} \\
\end{array}$ & 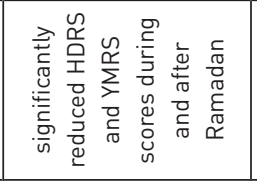 & 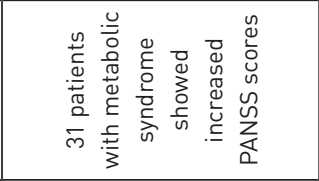 & 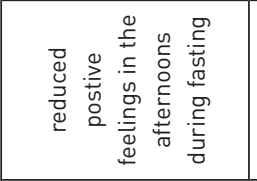 & 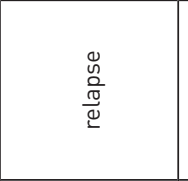 & 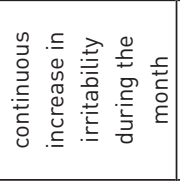 & 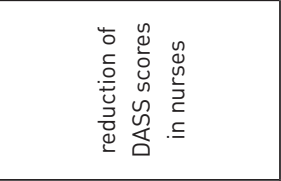 \\
\hline 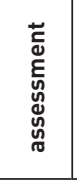 & 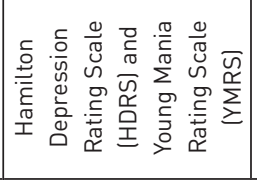 & 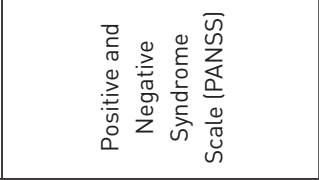 & 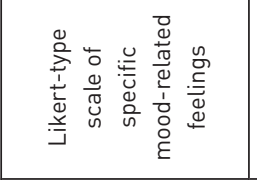 & 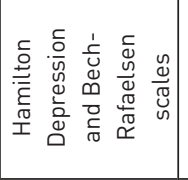 & 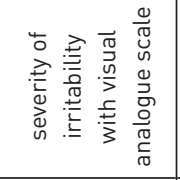 & 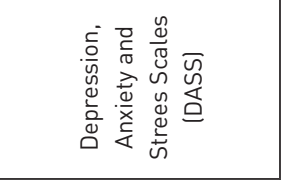 \\
\hline 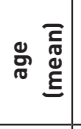 & 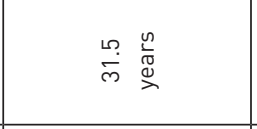 & 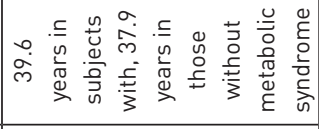 & 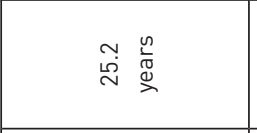 & 产 & 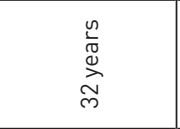 & 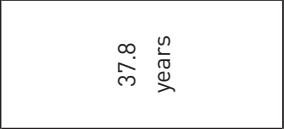 \\
\hline 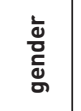 & 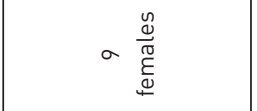 & 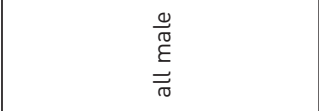 & 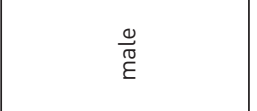 & 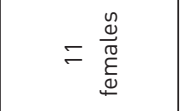 & $\stackrel{\frac{\varrho}{\varpi}}{\stackrel{\oplus}{\varepsilon}}$ & 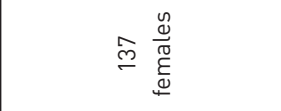 \\
\hline 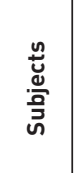 & 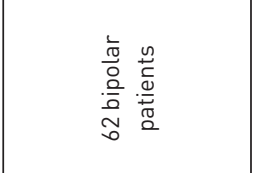 & 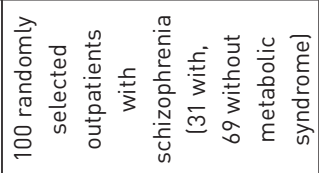 & 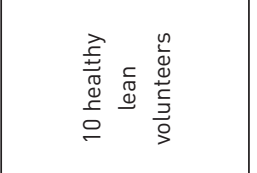 & 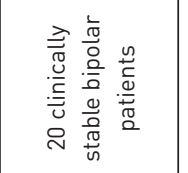 & 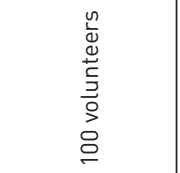 & 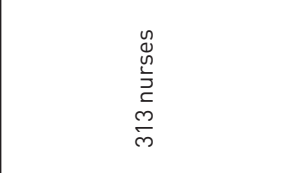 \\
\hline 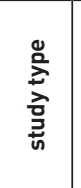 & 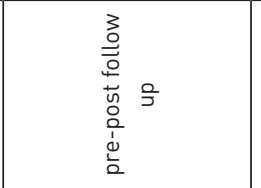 & 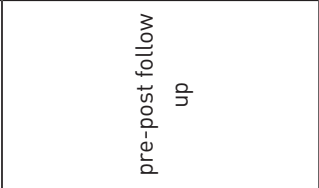 & 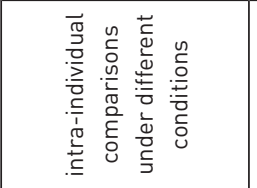 & 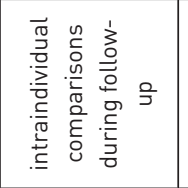 & 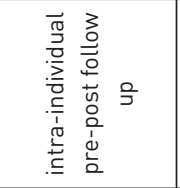 & 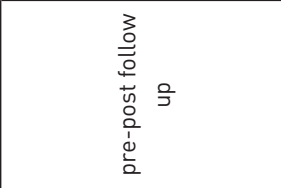 \\
\hline 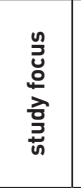 & 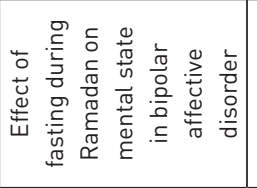 & 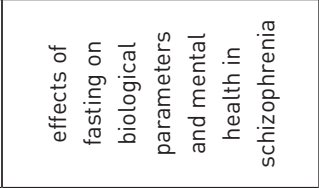 & 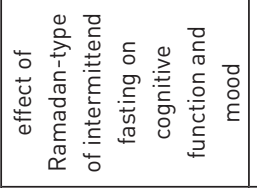 & 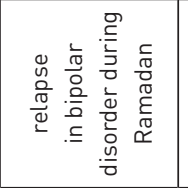 & 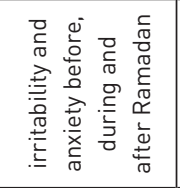 & 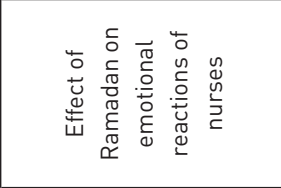 \\
\hline 咅 & 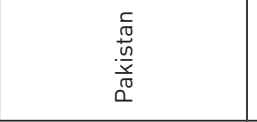 & 营 & 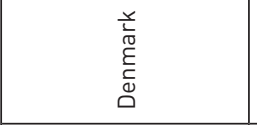 & 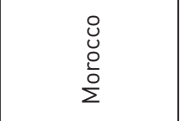 & 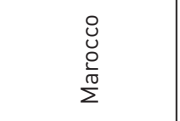 & $\stackrel{\text { 뜌 }}{\underline{\underline{T}}}$ \\
\hline 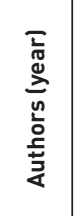 & 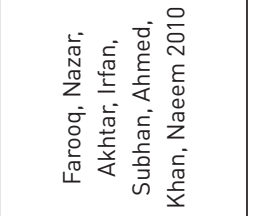 & 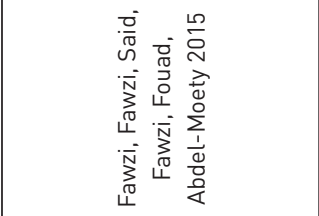 & 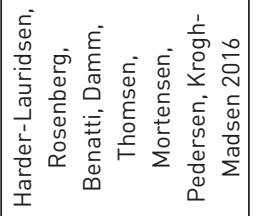 & 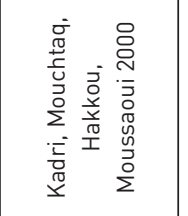 & 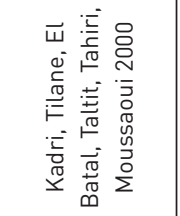 & 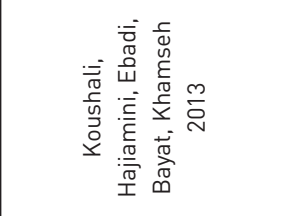 \\
\hline
\end{tabular}




\begin{tabular}{|c|c|c|c|c|c|}
\hline 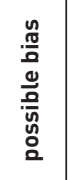 & 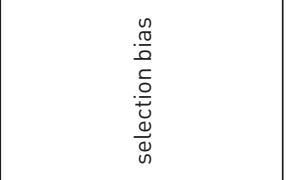 & 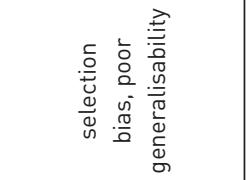 & 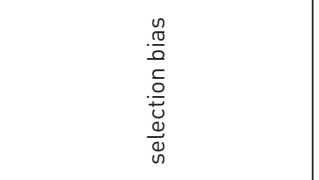 & 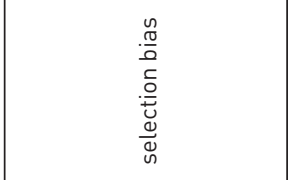 & 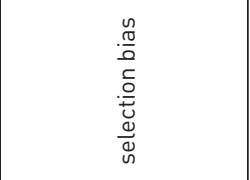 \\
\hline 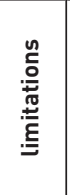 & 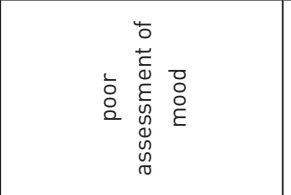 & 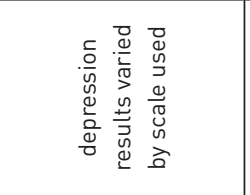 & 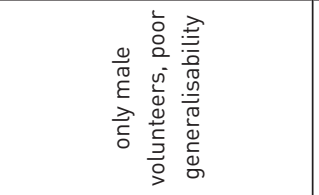 & 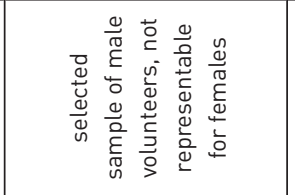 & 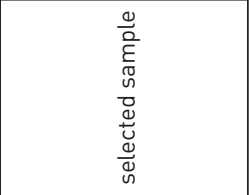 \\
\hline 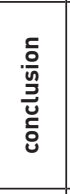 & 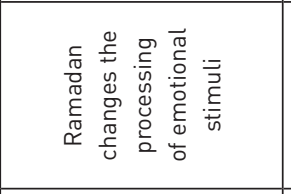 & 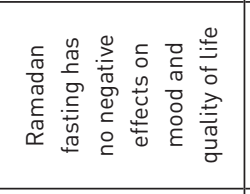 & 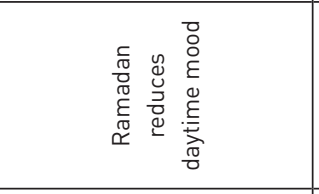 & 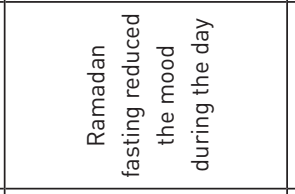 & 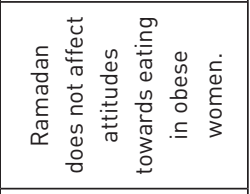 \\
\hline 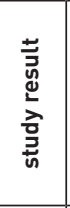 & 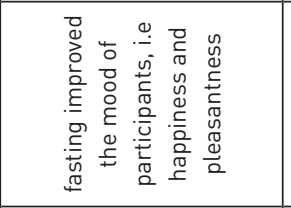 & 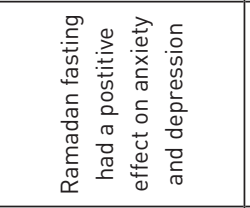 & 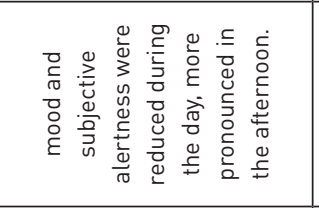 & 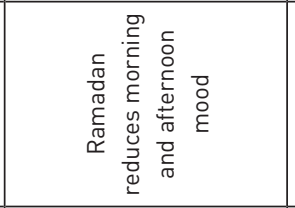 & 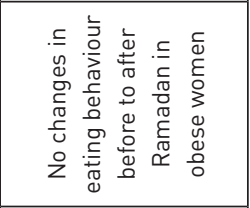 \\
\hline 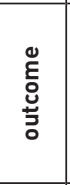 & 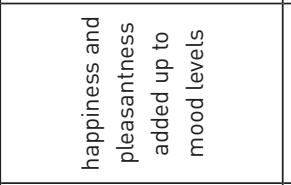 & 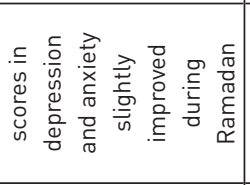 & 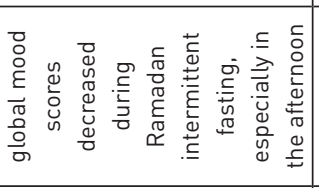 & 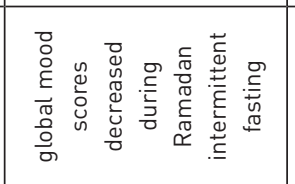 & 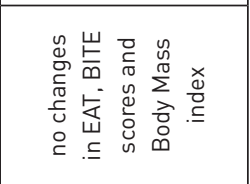 \\
\hline 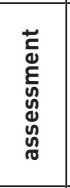 & 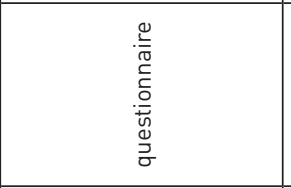 & 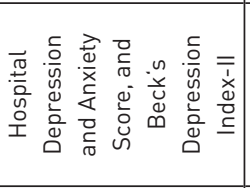 & 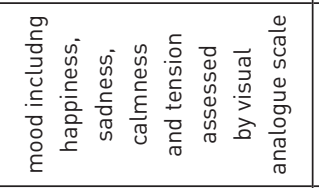 & 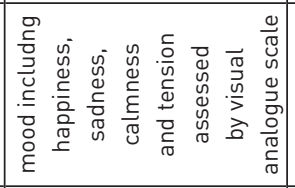 & 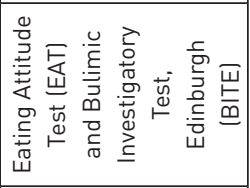 \\
\hline 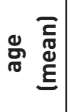 & 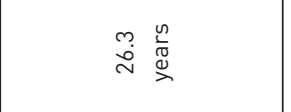 & 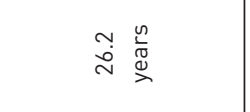 & 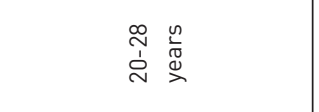 & 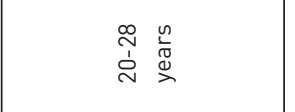 & 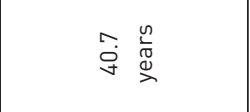 \\
\hline 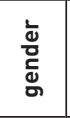 & $\sim \underset{\sim}{\stackrel{\mathscr{\vartheta}}{\pi}}$ & $\stackrel{\frac{0}{\tilde{\sigma}}}{\varepsilon}$ & $\frac{\stackrel{\oplus}{\leftrightarrows}}{\varepsilon}$ & $\stackrel{\frac{\omega}{\pi}}{\varepsilon}$ & 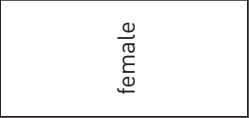 \\
\hline 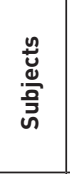 & 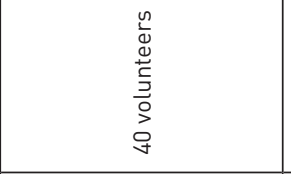 & 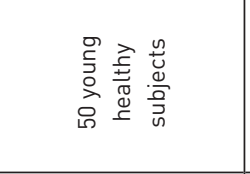 & 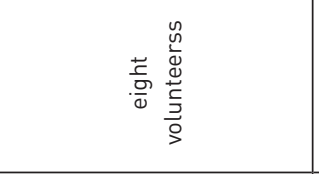 & 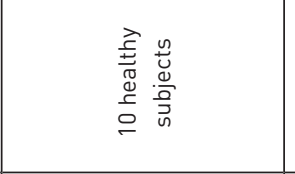 & 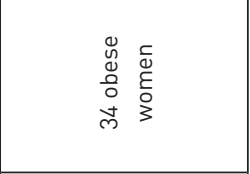 \\
\hline 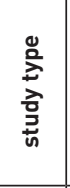 & 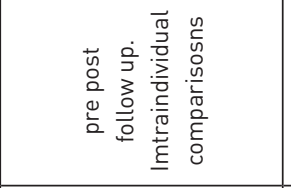 & 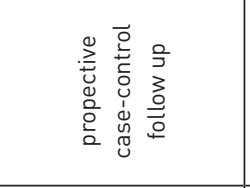 & 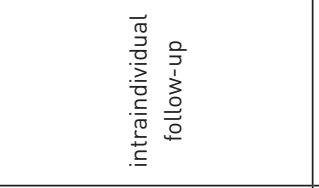 & 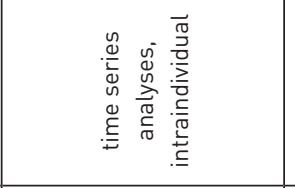 & 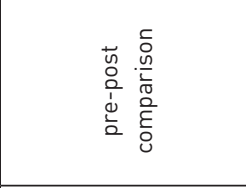 \\
\hline 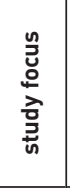 & 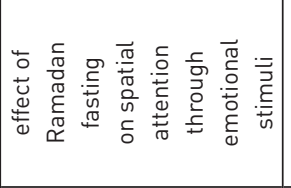 & 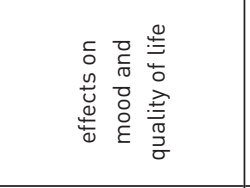 & 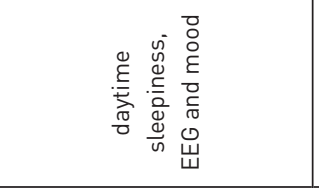 & 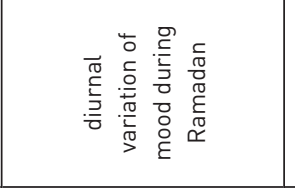 & 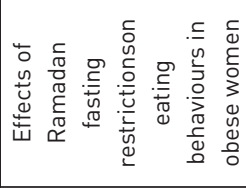 \\
\hline 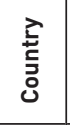 & $\begin{array}{l}\frac{\pi}{\sqrt{2}} \\
\frac{\pi}{\sqrt[\pi]{0}} \\
\frac{10}{2}\end{array}$ & 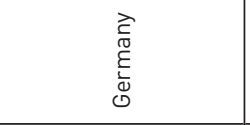 & 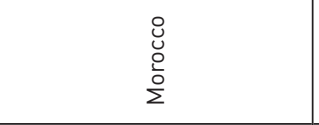 & 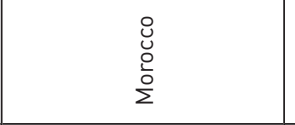 & 离 \\
\hline 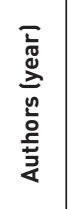 & 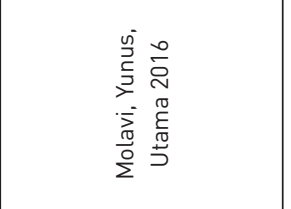 & 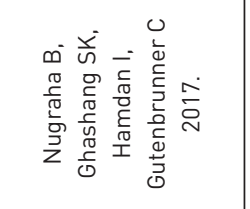 & 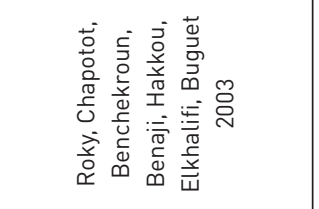 & 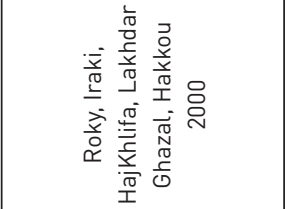 & 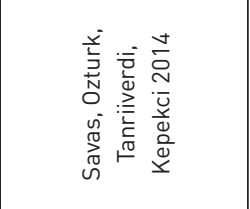 \\
\hline
\end{tabular}


was improved during Ramadan (Molawi et al. 2016). Officially reported para-suicides in Jordan were reduced during, but not after Ramadan (Daradkeh 1992).

In contrast, five studies reported negative effects: Ramadan fasting increased fatigue in nurses (Ovuyolu et al. 2016). Irritability and anxiety were increased in healthy male volunteers during Ramadan, with the effects being more pronounced in smokers (Kadri, Tilane et al. 2000). Roky et al. (2000, 2003) reported reduced subjective alertness and mood scores in 10 and 8 volunteers during the day throughout Ramadan. HarderLauridsen et al. (2016) also reported reduced mood levels in volunteers in the afternoon during Ramadan.

The contradiction in the outcomes of the studies cannot be neglected or easily explained by study type. These differences will make it necessary to properly assess under which circumstances positive or negative effects of Ramadan on mental health can be observed.

\section{Effects on mood in sports}

The effects of Ramadan on mental well-being in sports and physical activity were mostly positive, at least not negative: Sports performance and fatigue, but not mood, were reduced during compared to before Ramadan (Chennaoui et al. 2009, Chtourou et al. 2011). Aloui et al. (2015) saw increased sports performance in physical education students with music during Ramadan, but the different effects on enjoyment and anxiety were inconsistent with a motivational enhancement by music.

These positive outcomes may be related to the fact that the observed samples consist of young physically active subjects. In general, Aloui at al. (2016) recommend that judokas only try to lose weight before or after Ramadan.

\section{Effects in different physical disorders}

The effects of fasting on the mental health in people with some severe physical disorders were quite positive: Ramadan-related religious fasting did not cause depression or low mood in subjects with cancer-related stoma (Altuntas et al. 2013) and gastric sleeve surgery (Al-Ozairi et al. 2015). Mental health-related quality of life was even better after versus before Ramadan in patients with multiple sclerosis (Etemafidar et al. 2017).

In summary, there is some, even though limited, evidence that fasting can be performed well by subjects with some severe physical disorders.

\section{Effects on normal eating and eating disorders}

The publications on eating and eating disorders are positive in the way that eating disorders only seem to be triggered in a small subgroup of subjects with enhanced vulnerability to eating disorders: Akgul et al. (2014) observed an increase in hospital admissions during and shortly after Ramadan in a case series that may be caused by fasting induced triggering of eating problems in vulnerable adolescents. But Ramadan fasting did not affect attitudes towards eating in young volunteers (Erol et al. 2012) and obese women (Savas et al. 2014).

Thus, Ramadan fasting does not seem to change eating behaviour in general, but it may affect eating behaviour in few subjects vulnerable to eating disorders

\section{Effects in different mental health disorders}

There is limited evidence that the effects of Ramadan fasting on mental disorders is more harmful than beneficial: Ramadaninduced alcohol restriction may lead to acute alcohol withdrawal and finally acute paranoid symptoms (Al-Sinawi et al. 2008). There was no effect of Ramadan on mental health in patients with schizophrenia without metabolic syndrome; however, positive and negative symptoms of schizophrenia deteriorated in those with a metabolic syndrome (Fawzi et al. 2015). Disease-related psychopathology, that is, depression and manic symptoms, improved in patients with bipolar disorder during and after Ramadan (Farooq et al. 2010). However, Kadri, Mouchtaq et al. (2000) observed an increase of relapses during Ramadan. According to Eddahby et al. (2014), fasting during the Ramadan month increased the risk of relapse amongst patients with bipolar disorder by 2.77 fold in comparison to non-fasters.

Consequently, even though the psychopathology may reduce in bipolar disorder, there is a considerable excess risk of relapse during Ramadan. It may thus be advisable to recommend Ramadan fasting in subjects at risk of alcohol withdrawal, in patients with schizophrenia and metabolic syndrome and in patients with known bipolar disorder.

\section{DISCUSSION}

As expected, the majority of studies with relevance to mental health and Ramadan were performed in countries with a Moslem majority, such as Turkey, Morocco, Tunisia, Iran, Pakistan, Kuwait, Oman, Saudi Arabia and Malaysia. Only three studies were conducted in European countries, i.e. Denmark, France and Germany. 
The samples examined were the general population and healthy volunteers, subjects active in sports, subjects with severe physical disorders, subjects at risk of eating problems and patients with different mental health disorders.

The effects of Ramadan on the mood in healthy volunteers were positive in some studies but were negative in a few more others. Usually, the sample sizes were low, between 8 and 40 subjects, and mostly consisted of male volunteers.

The effects of Ramadan were positive in a larger sample of nurses, even though the fatigue levels were higher during Ramadan. The most positive and thus most relevant finding was the reduced number of para suicides during Ramadan in Jordan. This observation would need replication in other countries and samples to exclude a reporting and awareness bias during Ramadan. As self-harming behaviour is forbidden during Ramadan, there might be a specific risk of underreporting during this time.

In volunteers doing sports and physical activities, the effects of Ramadan are limited but were usually positive. Losing weight during Ramadan might be an increased challenge.

Ramadan did not reduce the mental well-being of subjects with cancer, gastric sleeve surgery and multiple sclerosis. Thus there should be no major medical objection concerning mental health if patients chose to fast during Ramadan.

Ramadan fasting did not have a major impact on the mental health in eating behaviour and eating disorders, even though there might be some triggering effects in vulnerable subjects.

Even though there is only rare evidence by a case report, it might be understandable that acute alcohol delirium and withdrawal may be a consequence of acute stopping of alcohol consumption. Ramadan fasting may not be recommended in subjects with schizophrenia and metabolic syndrome and in subjects with bipolar disorder because of a relevantly increased risk in relapse.

In summary, there is no major reason to recommend against fasting during Ramadan except in subjects with major psychiatric disorders. Even though some studies show some mood reduction and increased fatigue and anxiety, there is no study indicating that Ramadan fasting triggers new mental health problems or disorders.

Interestingly, there was no study that focussed on the effects of Ramadan fasting on subjects with or at risk of depression.
Future studies on the mental health during Ramadan should assess the effects in larger samples and in different countries. It might be relevant to assess the different social and environmental practices of fasting relating these to possible differences of outcome. It might be less interesting to assess the effects of Ramadan fasting on minute mood variations in small and highly selected samples of healthy male volunteers. These samples may have a high resilience to stress and may thus be less prone to changes in mood and mental well-being. It might be of more interest to identify the specific aspects of Ramadan, that is, fasting, fluid restriction, sleep delay and social gathering that may affect well-being in beneficial or harmful ways.

\section{LIMITATIONS}

This systematic review only included studies that relate to mental health, mental disorders and Ramadan. We did not focus on sleep, the effects of fasting on metabolism or the whole variety of physical disorders. As the studies available were quite variable in design and structure with low numbers of subjects, we were unable to provide substantial undisputable conclusions. The samples used made the individual studies prone to selection bias and thus reduce the generalisability of the studies.

\section{CONCLUSIONS}

The literature on mental health and Ramadan is mixed and patchy. Ramadan has a large influence on sleep, eating behaviour and social and religious interactions. These issues are all of great relevance to mental disorders. Some Moslems fast and some don't. However, it seems that the scientific potential of this large religiously motivated major social intervention has not been harvested neither for the understanding of mental disorders nor for the development of treatment. The possible results would not only be of relevance for the support of Moslems with mental health problems, but may also help increase the understanding of major social changes on mental health and relevant interventions on the well-being of the population.

An example may be smoking cessation interventions: Aveyard et al. (2011) recommend and discuss promoting smoking cessation through smoking reduction during Ramadan. However, there is no study that have used Ramadan fasting to initiate and assess smoking cessation outcomes. 
Thus, the interaction of mental health and Ramadan needs further research with larger samples, different populations from different environments and social backgrounds and with more appropriate diagnostic instruments measuring mental state and change. There could to be a deepened focus on the immense opportunities provided by this major social intervention for the understanding of sleep, fasting, social interactions, religion and, finally, mental health for the benefit of Moslem and non-Moslem patients with or at risk of mental health problems.

\section{ACKNOWLEDGEMENT}

I am very grateful to Meara Brooks and Mary Hickman for supporting the literature search.

\section{REFERENCES}

Akgul S, Derman 0, Kanbur NA. Fasting during Ramadan: a religious factor as a possible trigger or exacerbator for eating disorders in adolescents. Int J Eat Disord. 2014 Dec;47(8):905-10. doi: 10.1002/eat.22255.

Al-Ozairi E(1), Al Kandari J, AlHaqqan D, AlHarbi O, Masters Y, Syed AA. Obesity surgery and Ramadan: a prospective analysis of nutritional intake, hunger and satiety and adaptive behaviours during fasting. Obes Surg. 2015 Mar;25(3):523-9

Al-Sinawi H, Al-Adawi S, Al-Guenedi A. Ramadan fasting triggering koro-like symptoms during acute alcohol withdrawal: a case report from Oman.Transcult Psychiatry. 2008 Dec;45(4):695-704.

Aloui A, Chtourou H, Briki W, Tabben M, Chaouachi A, Souissi N, Shephard RJ, Chamari K. Rapid weight loss in the context of Ramadan observance: recommendations for judokas.

Biol Sport. 2016 Dec;33(4):407-413

Aloui A, Briki W, Baklouti H, Chtourou H, Driss T, Chaouachi A, Chamari K, Souissi N. Listening to Music during WarmingUp Counteracts the Negative Effects of Ramadan Observance on Short-Term Maximal Performance. PLoS One. 2015 Aug 24;10(8):e0136400

Altuntas YE, Gezen FC, Sahoniz T, Kement M, Aydin H, Sahin F, Okkabaz N, Oncel M. Ramadan fasting in patients with a stoma: a prospective study of quality of life and nutritional status. Ostomy Wound Manage. 2013;59(5):26-32.

Aveyard P, Begh R, Sheikh A, Amos A. Promoting smoking cessation through smoking reduction during Ramadan. Addiction. 2011;106(8):1379-80

Bahammam A, Alrajeh M, Albabtain M, Bahammam S, Sharif M. Circadian pattern of sleep, energy expenditure, and body temperature of young healthy men during the intermittent fasting of Ramadan. Appetite. 2010;54(2):426-9

Bahammam A. Does Ramadan fasting affect sleep? Int J Clin Pract. 2006 Dec;60(12):1631-7.

Bahammam AS, Almushailhi K, Pandi-Perumal SR, Sharif MM. Intermittent fasting during Ramadan: does it affect sleep? J Sleep Res. 2014 Feb;23(1):35-43. doi: 10.1111/jsr.12076. Epub 2013 Aug 12.

Chennaoui M, Desgorces F, Drogou C, Boudjemaa B, Tomaszewski A, Depiesse F,Burnat P, Chalabi H, Gomez-Merino D. Effects of Ramadan fasting on physical performance and metabolic, hormonal, and inflammatory parameters in middle-distance runners. Appl Physiol Nutr Metab. 2009 Aug;34(4):587-94. doi: 10.1139/H09-014.

Chtourou H, Hammouda O, Souissi H, Chamari K, Chaouachi A, Souissi N. The effect of Ramadan fasting on physical performances, mood state and perceived exertion in young footballers. Asian J Sports Med. 2011 Sep;2(3):177-85.

Daradkeh TK. Parasuicide during Ramadan in Jordan. Acta Psychiatr Scand. 1992 Sep;86(3):253-4.

Eddahby S, Kadri N, Moussaoui D Fasting during Ramadan is associated with a higher recurrence rate in patients with bipolar disorder. World Psychiatry. 2014 Feb;13(1):97. doi: 10.1002/wps.20113

Erol A, Baylan G, Yazici F. Do Ramadan fasting restrictions alter eating behaviours? Eur Eat Disord Rev. 2008 Jul;16(4):297-301. doi: 10.1002/erv.872.

Etemadifar M, Sayahi F, Alroughani R, Toghianifar N, Akbari M, Nasr Z. Effects of prolonged fasting on fatigue and quality of life in patients with multiple sclerosis. Neurol Sci. 2016 Jun;37(6):929-33 
Farooq S, Nazar Z, Akhtar J, Irfan M, Subhan F, Ahmed Z, Khan $E H$, Naeem F. Effect of fasting during Ramadan on serum lithium level and mental state in bipolar affective disorder. Int Clin Psychopharmacol. 2010 Nov;25(6):323-7

Fawzi MH, Fawzi MM, Said NS, Fawzi MM, Fouad AA, AbdelMoety H. Effect of Ramadan fasting on anthropometric, metabolic, inflammatory and psychopathology status of Egyptian male patients with schizophrenia. Psychiatry Res. 2015 Feb 28;225(3):501-8

Fond G, Macgregor A, Leboyer M, Michalsen A. Fasting in mood disorders: neurobiology and effectiveness. A review of the literature. Psychiatry Res. 2013 Oct 30;209(3):253-8

Harder-Lauridsen NM, Rosenberg A, Benatti FB, Damm JA, Thomsen C, Mortensen EL, Pedersen BK, Krogh-Madsen R. Ramadan model of intermittent fasting for $28 \hat{A} \mathrm{~d}$ had no major effect on body composition, glucose metabolism, or cognitive functions in healthy lean men. Nutrition. 2017 May;37:92-103

Kadri N, Tilane A, El Batal M, Taltit Y, Tahiri SM, Moussaoui D. Irritability during the month of Ramadan. Psychosom Med. 2000 Mar-Apr;62(2):280-5.

Kadri N, Mouchtaq N, Hakkou F, Moussaoui D Relapses in bipolar patients: changes in social rhythm? Int J Neuropsychopharmacol. 2000 Mar;3(1):45-49.

Koushali AN, Hajiamini Z, Ebadi A, Bayat N, Khamseh F. Effect of Ramadan fasting on emotional reactions in nurses. Iran J Nurs Midwifery Res. 2013 May;18(3):232-6.

Leiper JB, Junge A, Maughan RJ, Zerguini Y, Dvorak J. Alteration of subjective feelings in football players undertaking their usual training and match schedule during the Ramadan fast. J Sports Sci. 2008 Dec;26 Suppl 3:S55-69

Molavi M, Yunus J, Utama NP. The effect of Ramadan fasting on spatial attention through emotional stimuli. Psychol Res Behav Manag. 2016;9:105-14. doi: 10.2147/PRBM.S100495. eCollection 2016.

Nugraha B, Ghashang SK, Hamdan I, Gutenbrunner C. Effect of Ramadan fasting on fatigue, mood, sleepiness, and health-related quality of life of healthy young men in summer time in Germany: A prospective controlled study. Appetite. 2017;111:38-45
Ovayolu A, Ovayolu N, Tasan E. Does Ramadan Fasting Affect Fatigue in Nurses? Holist Nurs Pract. 2016 Jul-Aug;30(4):222-6

Roky R, Chapotot F, Benchekroun MT, Benaji B, Hakkou F, Elkhalifi $H$, Buguet A. Daytime sleepiness during Ramadan intermittent fasting: polysomnographic and quantitative waking EEG study. J Sleep Res. 2003 Jun;12(2):95-101.

Roky R, Iraki L, HajKhlifa R, Lakhdar Ghazal N, Hakkou F. Daytime alertness, mood, psychomotor performances, and oral temperature during Ramadan intermittent fasting. Ann Nutr Metab. 2000;44(3):101-7.

Savas E, Ozturk ZA, Tanriverdi D, Kepekci Y. Do Ramadan fasting restrictions alter eating behaviours in obese women? J Relig Health. 2014 Feb;53(1):135-40

Talib RA, Canguven 0, Al-Rumaihi K, Al Ansari A, Alani M. The effect of fasting on erectile function and sexual desire on men in the month of Ramadan. Urol J. 2015 Apr 29;12(2):2099-102.

Waterhouse J, Alkib L, Reilly T. Effects of Ramadan upon fluid and food intake, fatigue, and physical, mental, and social activities: a comparison between the UK and Libya. Chronobiol Int. 2008 Sep;25(5):697-724. 